\title{
Does the Chapman-Enskog expansion for viscous granular flows converge?
}

\author{
Andrés Santos \\ Departamento de Física, Universidad de Extremadura, E-06071 Badajoz, Spain
}

\begin{abstract}
This paper deals with the convergence/divergence issue of the Chapman-Enskog series expansion of the shear and normal stresses for a granular gas of inelastic hard spheres. From the exact solution of a simple kinetic model in the uniform shear and longitudinal flows, it is shown that (except in the elastic limit) both series converge and their respective radii of convergence increase with inelasticity. This paradoxical result can be understood in terms of the time evolution of the Knudsen number and the existence of a nonequilibrium steady state.
\end{abstract}

Keywords: Chapman-Enskog expansion, Granular gases, Rheological properties

PACS: $45.70 . \mathrm{Mg}, 05.20 . \mathrm{Dd}, 47.50 .-\mathrm{d}, 51.10 .+\mathrm{y}$

Introduction. In a Newtonian fluid the irreversible parts of the stress tensor $P_{i j}$ are linear in the velocity gradients $\nabla_{i} u_{j}$. For instance, if the flow is incompressible (i.e., $\nabla \cdot \mathbf{u}=0$ ), $P_{x y}=-\eta_{0} \partial u_{x} / \partial y$, where $\eta_{0}$ is the shear viscosity and it has been assumed that $\partial u_{y} / \partial x=0$. Analogously, in a compressible flow characterized by a rate-of-strain tensor $\nabla_{i} u_{j}=\left(\partial u_{x} / \partial x\right) \delta_{i x} \delta_{j x}$ one has $P_{x x}=p-(4 / 3) \eta_{0} \partial u_{x} / \partial x$, where $p$ is the hydrostatic pressure and the bulk viscosity has been neglected (as happens in the low-density limit). In the case of a dilute gas, Newton's law is derived from the Boltzmann equation under the assumption that the characteristic hydrodynamic length $(L)$ associated with the gradients of density $(n)$, temperature $(T)$, or flow velocity $(\mathbf{u})$ is much larger than the mean free path $\ell$ of the gas particles, i.e., $\ell \ll L$. More in general, the Chapman-Enskog (CE) method expresses the solution of the Boltzmann equation as an expansion in powers of the Knudsen number $|\mu| \equiv \ell / L[1]$. The leading terms in the CE expansion yield the Navier-Stokes constitutive equations and provide explicit expressions for the transport coefficients (like the shear viscosity $\eta_{0}$ ).

An important basic question is the nature (convergent versus divergent) of the CE expansion. To isolate the problem, let us consider the following subclasses of the full CE series:

$$
P_{x y}=-\sum_{k=0}^{\infty} \eta_{k}\left(\partial u_{x} / \partial y\right)^{2 k+1}, \quad P_{x x}=p-\frac{4}{3} \sum_{k=0}^{\infty} \eta_{k}^{\prime}\left(\partial u_{x} / \partial x\right)^{k+1}
$$

where $\eta_{0}=\eta_{0}^{\prime}$ is the shear viscosity, $\eta_{1}^{\prime}$ is a Burnett coefficient, $\eta_{1}$ and $\eta_{2}^{\prime}$ are super-Burnett coefficients, and so on. The two full CE series of $P_{x a}$ (with $a=y$ and $a=x$, respectively) reduce to the partial series (1) if (and only if) $\nabla_{i} u_{j}=\dot{\gamma}_{x a} \delta_{i a} \delta_{j x}$ and $\nabla n=\nabla T=\nabla \dot{\gamma}_{x a}=\mathbf{0}$, i.e., the only nonzero hydrodynamic gradient is a uniform shear or longitudinal rate $\dot{\gamma}_{x a} \equiv \partial u_{x} / \partial a$. These are precisely the conditions characterizing two well-defined physical states: the uniform shear flow (USF, $a=y$ ) [2, 3, 4] and the uniform longitudinal flow (ULF, $a=x$ ) [5, 6]. In both states the characteristic hydrodynamic length is $L \sim \sqrt{2 T / m} /\left|\dot{\gamma}_{x a}\right|$ (where $m$ is the mass of a particle and the temperature is measured in energy units), so that the Knudsen number becomes the absolute value of $\mu \equiv \dot{\gamma}_{x a} / v$, where $v \sim \sqrt{2 T / m} / \ell$ is a characteristic collision frequency. Thus, the CE expansions (1) can be recast into the dimensionless forms

$$
P_{x y} / p=-\mu F_{x y}(\mu), \quad P_{x x} / p=1-\frac{4}{3} \mu F_{x x}(\mu), \quad F_{x y}(\mu) \equiv \sum_{k=0}^{\infty} c_{k} \mu^{2 k}, \quad F_{x x}(\mu) \equiv \sum_{k=0}^{\infty} c_{k}^{\prime} \mu^{k},
$$

where $c_{k} \equiv\left(\eta_{k} / p\right) v^{2 k+1}$ and $c_{k}^{\prime} \equiv\left(\eta_{k}^{\prime} / p\right) v^{k+1}$. Despite the simple definitions of the USF and the ULF, no exact solution of the nonlinear Boltzmann equation is known for those states. However, the problem becomes solvable in the framework of the Bhatnagar-Gross-Krook (BGK) model kinetic equation [3, 6] and the solutions show that, except for Maxwell molecules, the CE expansions (11) are divergent [4, 6].

So far we have implicitly assumed conventional gases made of particles that collide elastically. What about the case of granular gases? A granular gas is a large collection of macroscopic particles which collide inelastically and are 
maintained in a fluidized state. It can be conveniently modeled as a gas of smooth inelastic hard spheres characterized by a constant coefficient of normal restitution $\alpha<1$ [7]. For such a model, the CE method has been applied to the inelastic Boltzmann equation and the Navier-Stokes transport coefficients have been derived [8]. Taking into account that the nonlinear subclasses (1) of the full CE expansions, as said before, do not converge when the gas is made of elastic hard spheres $(\alpha=1)[4,6]$, and considering the inherently non-Newtonian nature of the steady USF of granular gases [9], it seems intuitive to expect that the CE series (11) are also divergent in the case of inelastic hard spheres. However, it turns out that, paradoxically, the series (1) do converge in the inelastic case, the radii of convergence increasing with increasing inelasticity [10, 11].

Rheological properties. In the USF, the mass and momentum balance equations yield constant density $n$ and shear rate $\dot{\gamma}_{x y}$. However, $n(t)=n(0) /\left[1+\dot{\gamma}_{x x}(0) t\right]$ and $\dot{\gamma}_{x x}(t)=\dot{\gamma}_{x x}(0) /\left[1+\dot{\gamma}_{x x}(0) t\right]$ in the ULF, so that $\dot{\gamma}_{x x}>0$ corresponds to an expansion of the gas, while $\dot{\gamma}_{x x}<0$ corresponds to a condensation phenomenon [5, 6]. In either case the ratio $\dot{\gamma}_{x a}(t) / n(t)$ is constant. As for the energy balance equation of inelastic hard spheres in the USF and ULF, one has

$$
\partial_{t} T(t)=-\left(2 \dot{\gamma}_{x a} / 3 n\right) P_{x a}(t)-\zeta(t) T(t),
$$

where $\zeta$ is the so-called cooling rate [7], which is approximately given by $\zeta=\frac{5}{12}\left(1-\alpha^{2}\right) v_{0}, v_{0} \propto n \sqrt{T}$ being an effective collision frequency for elastic spheres. The cooling term on the right-hand side of Eq. (3) competes with the viscous heating term in the USF $\left(\dot{\gamma}_{x y} P_{x y}<0\right)$ and in the ULF with $\dot{\gamma}_{x x}<0$ (note that $P_{x x}>0$ ), so that, depending on the initial state, the temperature either grows or decreases with time until a steady state is eventually reached [9, 11, 12], except in the elastic case $(\zeta=0)$. On the other hand, in the ULF with $\dot{\gamma}_{x x}>0$ both terms on the right-hand side of Eq. (3) represent cooling effects, so $T(t) \rightarrow 0$ and no steady state exists.

In order to analyze in detail the CE series (1), it is convenient to consider the following BGK-like kinetic model [13] of the inelastic Boltzmann equation:

$$
\left(\partial_{t}+\mathbf{v} \cdot \nabla\right) f=-v\left(f-f_{0}\right)+(\zeta / 2) \partial_{\mathbf{v}} \cdot[(\mathbf{v}-\mathbf{u}) f],
$$

where $f$ is the velocity distribution function, $f_{0}$ is the local version of the homogeneous cooling state distribution [7], and $v=\frac{1+\alpha}{2} v_{0}$ is an effective collision frequency, so that $\varepsilon \equiv \zeta / v=\frac{5}{6}(1-\alpha)$. Taking moments in Eq. (4) one gets

$$
\mathrm{USF}:\left\{\begin{array}{l}
\partial_{t} P_{x y}=-\dot{\gamma}_{x y} P_{y y}-(v+\zeta) P_{x y}, \quad \mathrm{ULF}: \partial_{t} P_{x x}=v p-\left(v+\zeta+3 \dot{\gamma}_{x x}\right) P_{x x}, \\
\partial_{t} P_{y y}=v p-(v+\zeta) P_{y y},
\end{array}\right.
$$

where $p=n T$. Equations (3) and (5) constitute a closed set of equations for the evolution of $\left\{T, P_{x y}, P_{y y}\right\}$ (USF) or $\left\{T, P_{x x}\right\}$ (ULF). To describe the non-Newtonian hydrodynamic regime we must focus on the nonlinear dependence of the scaled quantities $F_{x y}(\mu(t)) \equiv-P_{x y}(t) / p(t) \mu(t)$ and $F_{x x}(\mu(t)) \equiv-(3 / 4)\left[P_{x x}(t) / p(t)-1\right] / \mu(t)$ as functions of $\mu(t) \equiv \dot{\gamma}_{x a}(t) / v(t) \propto 1 / \sqrt{T(t)}$. Elimination of time in favor of $\mu$ in Eqs. (3) and (5) yields a single second-order ordinary differential equation for $F_{x y}(\mu)$ [10] and a single first-order ordinary differential equation for $F_{x x}(\mu)$ [11].

Results and discussion. The numerical solutions for a gas of elastic hard spheres $(\alpha=1)$ and for a gas of highly inelastic hard spheres $(\alpha=0.5)$ are plotted in Fig. 1 a). The arrows on the curves indicate the direction followed by the time evolution of $\mu(t)$. In the inelastic case $(\alpha<1, \varepsilon \neq 0)$ the evolution leads to the steady-state point $\mu_{s}= \pm \sqrt{3 \varepsilon / 2}(1+\varepsilon), F_{x y}\left(\mu_{s}\right)=1 /(1+\varepsilon)^{2}$ for the USF and $\mu_{s}=-3 \varepsilon(1+\varepsilon) / 2(1+3 \varepsilon), F_{x x}\left(\mu_{s}\right)=(1+3 \varepsilon) /(1+\varepsilon)^{2}$ for the ULF (provided that $\dot{\gamma}_{x x}<0$; otherwise, $\mu(t) \rightarrow \infty$ ). On the other hand, in the elastic case $(\alpha=1, \varepsilon=0)$ the temperature monotonically increases with time in the USF and in the ULF with $\dot{\gamma}_{x x}<0$ and thus the Knudsen number asymptotically vanishes, i.e., $\mu(t) \rightarrow 0$. The dependence of $\mu_{s}$ on $\alpha$ is shown in Figure1(b).

According to the preceding discussion, the zero Knudsen number value $(\mu=0)$ is a "repeller" of the time evolution of $\mu(t)$ both for USF and ULF in the inelastic case. For elastic collisions, however, the state $\mu=0$ is an "attractor" of $\mu(t)$ (except in the ULF with $\dot{\gamma}_{x x}>0$ ). Expressed in other terms, the fixed point $\mu=0$ is unstable against any USF or ULF perturbation, no matter how weak it is, if the particles are inelastic. On the other hand, $\mu=0$ is stable for elastic collisions in the USF and in the ULF with $\dot{\gamma}_{x x}<0$. Since the CE expansions (1) and (2) are carried out about (and measure the departure from) the reference homogeneous state $(\mu=0)$, it follows that the CE series diverge if $\lim _{t \rightarrow \infty} \mu(t)=0$ (elastic case), whereas they converge if $\mu(t)$ flees from $\mu=0$ (inelastic case). In the latter situation, in addition, the radius of convergence must coincide with the steady-state value $\mu_{s}$. Consequently, one must have $\lim _{k \rightarrow \infty}\left|c_{k} / c_{k+1}\right|=\mu_{s}^{2}$ and $\lim _{k \rightarrow \infty}\left|c_{k}^{\prime} / c_{k+1}^{\prime}\right|=\left|\mu_{s}\right|$. This expectation is confirmed by an exact evaluation of the 

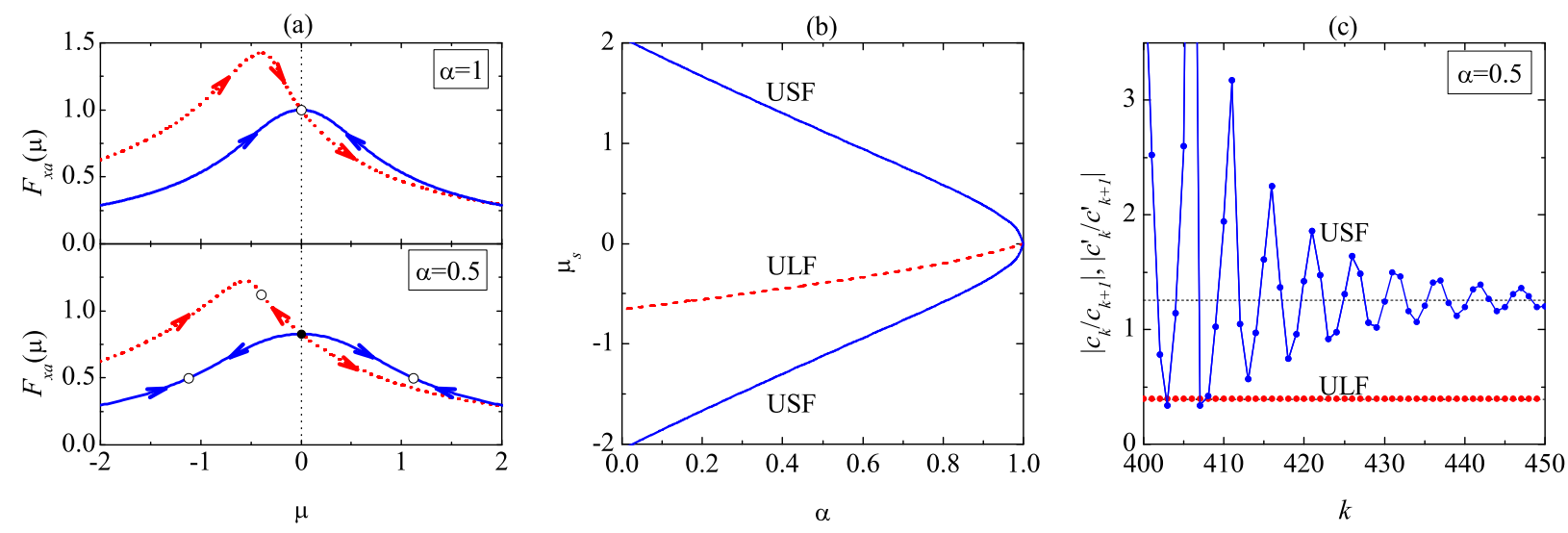

FIGURE 1. (a) Plot of $F_{x y}(\mu)$ (solid lines) and $F_{x x}(\mu)$ (dotted lines) for $\alpha=1$ and $\alpha=0.5$; the open circles denote the steadystate points. (b) Dependence of the steady-state value $\mu_{s}$ on the coefficient of restitution $\alpha$. (c) Ratios $\left|c_{k} / c_{k+1}\right|$ and $\left|c_{k}^{\prime} / c_{k+1}^{\prime}\right|$ for $\alpha=0.5$; the horizontal dotted lines represent $\mu_{s}^{2}$ and $\left|\mu_{s}\right|$, respectively.

coefficients $c_{k}$ and $c_{k}^{\prime}$ for $k \leq 450$, as illustrated by Fig. 1(c) for $\alpha=0.5$. In mathematical terms, the nonlinear viscosity functions $F_{x a}(\mu)$ possess a singularity located at $\mu=0$ in the elastic case and at $\mu=\mu_{s} \neq 0$ in the inelastic case.

To conclude, the paradoxical regularization by inelasticity of the CE series (1) is directly related to the time evolution of the Knudsen number $|\mu(t)|$, and hence of the temperature, Eq. (3), and thus it is not an artifact of the kinetic model (4). Also, the convergence or divergence of the series (1) does not depend on whether the system actually is in the USF, the ULF, or in any other state. The advantage of the USF and ULF is that the full CE series of the shear and normal stresses reduce to the partial series (1), thus allowing one to explore their character in a detailed way.

\section{ACKNOWLEDGMENTS}

This work has been supported by the Ministerio de Educación y Ciencia (Spain) through Grant No. FIS2007-60977 (partially financed by FEDER funds) and by the Junta de Extremadura (Spain) through Grant No. GRU08069.

\section{REFERENCES}

1. S. Chapman and T. G. Cowling, The Mathematical Theory of Non-uniform Gases, Cambridge U.P., Cambridge, 1970.

2. A. W. Lees and S. F. Edwards, J. Phys. C: Solid State Phys. 5, 1921-1929 (1972).

3. V. Garzó and A. Santos, Kinetic Theory of Gases in Shear Flows. Nonlinear Transport, Kluwer, Dordrecht, 2003.

4. A. Santos, J. J. Brey, and J. W. Dufty, Phys. Rev. Lett. 56, 1571-1574 (1986); A. Santos and J. J. Brey, Physica A 174, 355-390 (1991).

5. A. N. Gorban and I. V. Karlin, Phys. Rev. Lett. 77, 282-285 (1996); I. V. Karlin, G. Dukek, and T. F. Nonnenmacher, Phys. Rev. E 55, 1573-1576 (1997).

6. A. Santos, Phys. Rev. E 62, 6597-6607 (2000).

7. N. Brilliantov and T. Pöschel, Kinetic Theory of Granular Gases, Oxford U. P., Oxford, 2004; C. S. Campbell, Annu. Rev. Fluid Mech. 22, 57-92 (1990); J. W. Dufty, J. Phys.: Condens. Matt. 12 A47-A56 (2000); I. Goldhirsch, Annu. Rev. Fluid Mech. 35, 267-293 (2003).

8. J. J. Brey, J. W. Dufty, C. S. Kim, and A. Santos, Phys. Rev. E 58, 4638-4653 (1998); V. Garzó and J. W. Dufty, Phys. Rev. E 59, 5895-5911 (1999); Phys. Fluids 14, 1476-1490 (2002); V. Garzó, J. W. Dufty, and C. M. Hrenya, Phys. Rev. E 76, 031303-1-27 (2007); V. Garzó, C. M. Hrenya, and J. W. Dufty, Phys. Rev. E 76, 031304-1-20 (2007); S. H. Noskowicz, O. Bar-Lev, D. Serero, and I. Goldhirsch, Europhys. Lett. 79, 60001-1-6 (2007).

9. A. Santos, V. Garzó, and J. W. Dufty, Phys. Rev. E 69, 061303-1-10 (2004).

10. A. Santos, Phys. Rev. Lett. 100, 078003-1-4 (2008).

11. A. Santos, "Longitudinal viscous flow in granular gases," to appear in Rarefied Gas Dynamics: 26th International Symposium on Rarefied Gas Dynamics, AIP Conference Proceedings.

12. A. Astillero and A. Santos, Europhys. Lett. 78, 24002-1-6 (2007).

13. J. J. Brey, J. W. Dufty, and A. Santos, J. Stat. Phys. 97, 281-322 (1999). 